7. Corvus Pharmaceuticals, Inc. Corvus Pharmaceuticals Provides Business Update and Reports Second Quarter 2020 Financial Results. 30 July 2020. [https://corvuspharma.gcs-web.com/news-releases/news-release-details/corvus-pharmaceuticalsprovides-business-update-and-reports-4]

http://dx.doi.org/10.1136/jitc-2020-SITC2020.0476

\section{DEEP LEARNING TO DRIVE COVID-19 RAPID DRUG REPURPOSING}

Sarah Kolitz * , Jason Kim, Jenny Zhang, Yoonjeong Cha, Sailaja Battula, Rebecca Kusko, Rajaraman Krishnan, Benjamin Zeskind, Howard Kaufman. Immuneering Corp., Cambridge, MA, USA

Background COVID-19 is a global public health crisis with no effective therapeutic strategies or vaccines available. The disease is caused by the SARS-CoV-2 virus, a novel coronavirus that enters cells through the ACE2 receptor. To rapidly identify existing drugs that might preferentially bind to the ACE2 receptor we sought to use an artificial intelligence platform to evaluate $\sim 3,000$ known drugs in the FDA approved drug library (Selleckchem).

Methods Fluency is a quantitative structure-activity relationship (QSAR) deep learning-based platform that evaluates small molecule drug binding to protein targets. All drug structures from the FDA approved library were evaluated for binding to the ACE2 receptor and re-filtered for preferential ACE2 vs. ACE1 receptor binding. Top hits were evaluated for specificity by predicting binding across the human proteome and filtered by evaluating rankings from each of two models along with average ranks and combined scores from both models. The drugs were then evaluated for classification, potential availability and prioritized for in vitro validation. Selected compounds were screened using a high-throughput SARS-CoV-2 cell-based assay as described previously (Jonsson et al. J Biomol Screen 2007 12: 33. DOI: 10.1177/1087057106296688). Plates are quality-controlled in each run using $\mathrm{Z}$ score and CV statistics. Positive controls consisting of cells only and negative controls consisting of virus were used to normalize the data. Individual drugs are added to each plate at a single dose with at least four doses tested. For titer reduction assays, VeroE6 cells are infected with virus at MOI of 0.1 for one hour to promote adsorption. After two days, the supernatant is harvested and the amount of virus in each well is measured using TCID50 or plaque assay.

Results We identified 25 top drugs that were predicted to bind to ACE2 receptors and could theoretically block SARSCoV-2 cell entry. Of these drugs, we prioritized 12 drugs for validation covering multiple pharmacologic classes and after assessing drug availability (table 1). They included an ALK/ EGFR inhibitor, JAK inhibitor, two electrolyte channel inhibitors, lan antibiotic, and several anti-viral drugs, ACE inhibitors and anticoagulants. Validation studies are in progress and viral inhibition and titer reduction data will be presented.

Conclusions Our data show that machine learning platforms can be used to rapidly identify existing drugs that may have activity against SARS-CoV-2 infection. This hybrid computational and experimental approach enables rapid discovery of drugs for clinical testing against COVID-19 and other emerging human diseases.

Acknowledgements We would like to thank Dr. Colleen Jonsson and Dr. Jeremy Smith at Oak Ridge National Laboratories and the University of Tennessee Regional Biocontainment Laboratory for assistance with in vitro validation studies.

Trial Registration N/A
Ethics Approval N/A

Consent N.A

http://dx.doi.org/10.1136/jitc-2020-SITC2020.0477

\section{TRANSLATION OF A THERAPEUTIC NEOANTIGEN VACCINE WORKFLOW TO SARS-COV-2 VACCINE DEVELOPMENT}

${ }^{1}$ Christof Smith*, 'Sarah Entwistle, ${ }^{1}$ Caryn Willis, ${ }^{1}$ Steven Vensko, ${ }^{1}$ Wolfgang Beck, ${ }^{1}$ Jason Garness, ${ }^{1}$ Maria Sambade, ${ }^{1}$ Eric Routh, ${ }^{1}$ Kelly Olsen, ${ }^{1}$ Brandon Carpenter, ${ }^{1}$ Kaylee Gentry, ${ }^{1}$ Maria Fadri, ${ }^{1}$ Misha Fini, ${ }^{1}$ Amber Washington, ${ }^{2} J u l i a$ Kodysh ${ }^{2}$ Timothy O'Donnell, ${ }^{3}$ Carsten Haber, ${ }^{3}$ Kirsten Heiss, ${ }^{3}$ Volker Stadler, ${ }^{4}$ Erik Garrison, ${ }^{5}$ Oliver Grant, ${ }^{5}$ Robert Woods, ${ }^{1}$ Mark Heise, ${ }^{1}$ Benjamin Vincent, ${ }^{1}$ Alexander Rubinsteyn. 'UNC Chapel Hill, Chapel Hill, NC, USA; ${ }^{2}$ Icahn School of Medicine at Mount Sinai, New York, NY, USA; ${ }^{3}$ PEPperPRINT GmbH, Heidelberg, Germany; ${ }^{4}$ University of California, Santa Cruz, Santa Cruz, CA, USA; ${ }^{5}$ University of Georgia, Athens, GA, USA

Background There is an urgent need for a vaccine with efficacy against SARS-CoV-2. We hypothesize that peptide vaccines containing epitope regions optimized for concurrent $\mathrm{B}$ cell, CD4 $+\mathrm{T}$ cell, and CD8 $+\mathrm{T}$ cell stimulation would drive both humoral and cellular immunity with high specificity, potentially avoiding undesired effects such as antibody-dependent enhancement (ADE) (figure 1). Leveraging methods initially developed for prediction of tumor-specific antigen targets, we combine computational prediction of $\mathrm{T}$ cell epitopes, recently published $\mathrm{B}$ cell epitope mapping studies, and epitope accessibility to select candidate peptide vaccines for SARS-CoV-2 (figure 2).

Methods SARS-CoV-2 HLA-I and HLA-II ligands were predicted using multiple MHC binding prediction software. T cell vaccine candidates were further refined by predicted immunogenicity, viral source protein abundance, sequence

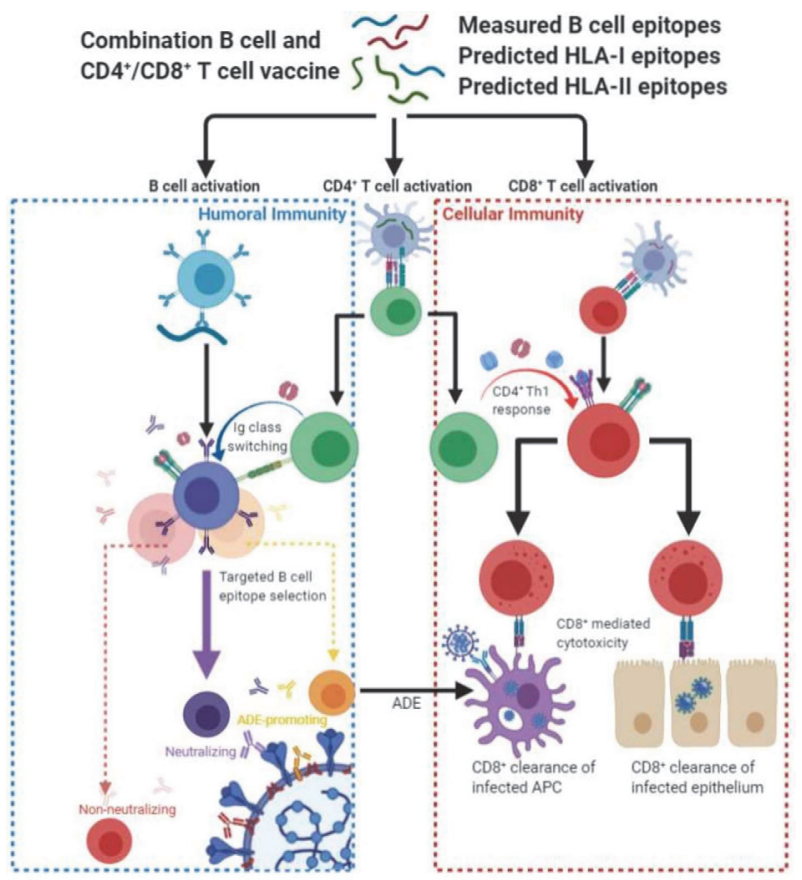

Abstract 478 Figure 1 Summary of combination CD4+/CD8+ T cell and B cell SARS-CoV-2 peptide vaccine. Humoral immunity (blue dashed box) is targeted through B cell and HLA-II epitopes, aimed at viral neutralization while avoiding non-neutralizing and ADE promoting targets. Cellular immunity (red dashed box) is targeted through HLA-I and HLA-II epitopes, aimed to clear virally infected cells 